EPJ Web of Conferences 92,02090 (2015)

DOI: 10.1051/epjconf/ 20159202090

C Owned by the authors, published by EDP Sciences, 2015

\title{
Hub and shroud fillets influence on the radial compressor stage efficiency
}

\author{
Tomáš Syka ${ }^{1, a}$, Richard Matas ${ }^{1}$ and Jindrich Kňourek ${ }^{1}$ \\ ${ }^{1}$ University of West Bohemia, New Technologies - Research centre, 30614 Pilsen, Czech Republic
}

\begin{abstract}
Article describes numerical simulations of an air flow in the radial compressor stage in the NUMECA CFD software. During the tasks evaluating the stepped and straight impeller seals and hub and shroud fillets influence on working characteristics and flow field was observed. Also the CFD results comparison with results from the empiric design tool and the measurement is described.
\end{abstract}

\section{Introduction}

This article deals with the influence of the presence of impeller blades hub and shroud fillets in the radial compressor stage model on the stage pressure ratio and the efficiency characteristics. During the design of modern blade machines 1D design computation tools are commonly used. These tools are able to estimate working characteristics of future stages, but they have certain limits. It is still only estimated solution, which is needed to confirm by the experimental data or complex CFD simulations. The big disadvantage of CFD simulations is necessary simplification of the geometrical domain because of computational resources. Due to this certain inaccuracy is always presented.

The main goal of this article is to compare results of the measurement on the real compressor stage and results from CFD simulations. We would like to find out how much the presence of the impeller fillets affects the compressor working characteristics shape. This is important because the fillets presence should cover differences between measured and computed CFD data.

Compressor stages numerical simulations were solved in NUMECA FINE/Turbo software.

\section{Computed compressor stage}

This stage is specific for the vaneless diffuser and for the shape of the channel intake area. An intake area is axial but changes to the radial direction and substitutes return channel of the previous stage in limited size. Impeller blades are 3D shaped and impeller seals are considered.

Stage simulations were solved only for one revolutions of the impeller - $13568 \mathrm{rpm}$. The stage works with relative medium mass flow coefficient and pressure ratios.

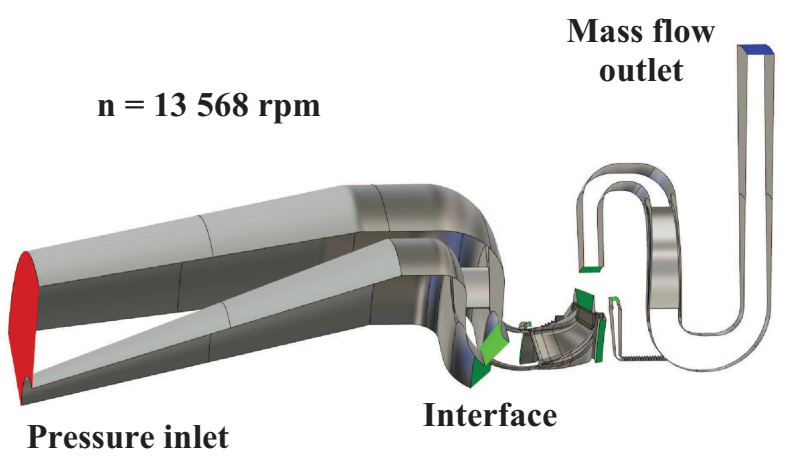

Figure 1. The radial stage model.

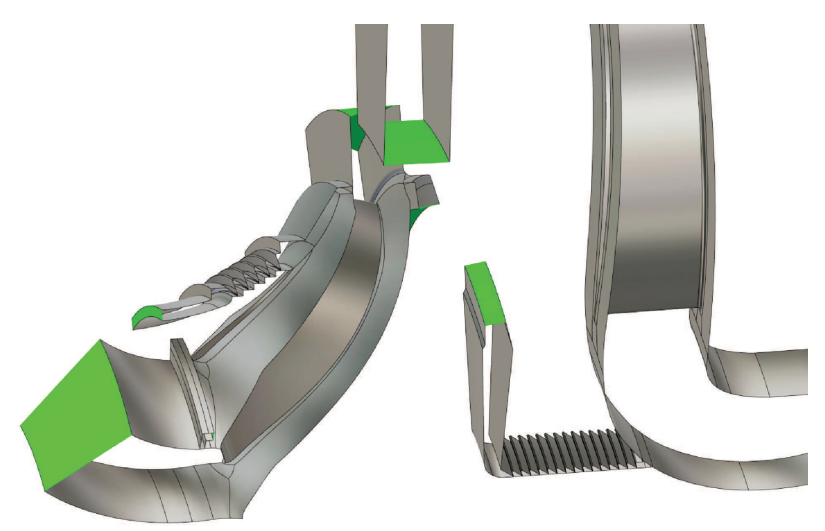

Figure 2. The detail of stage impeller with seals.

\subsection{Geometry and mesh creation}

The computational grid was created in the NUMECA AutoGrid software. The grid consists only of hexahedral

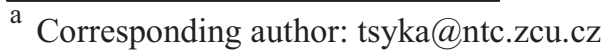


cells and is block structured because of the computational time reduction.

As a base for computational grid creation the blades geometry, border curves of the stage channel and the impeller seals geometry are required from the complete 3D CAD stage model. This step was done in the ANSA software. ANSA is the suitable tool for the work with general surfaces and curves. For these and other benefits ANSA is being used in the automotive industry for creating and modifying of very complex geometry models. For the creation of the channel grid only one blade from each row is needed.

\subsubsection{Base geometric stage model with seals and without impeller blades fillets}

In case of the geometry model variant without impeller hub and shroud fillets the mesh creation is quite automatic. After import of the prepared geometry into NUMECA/AutoGrid environment the definition of border curves is required. It means assigning certain curves to the hub and the shroud of the channel. Similar process is used for the definition of blades. After setting up of several parameters like the number of blades and the number of grid elements generating of the block structured computational grid is possible.

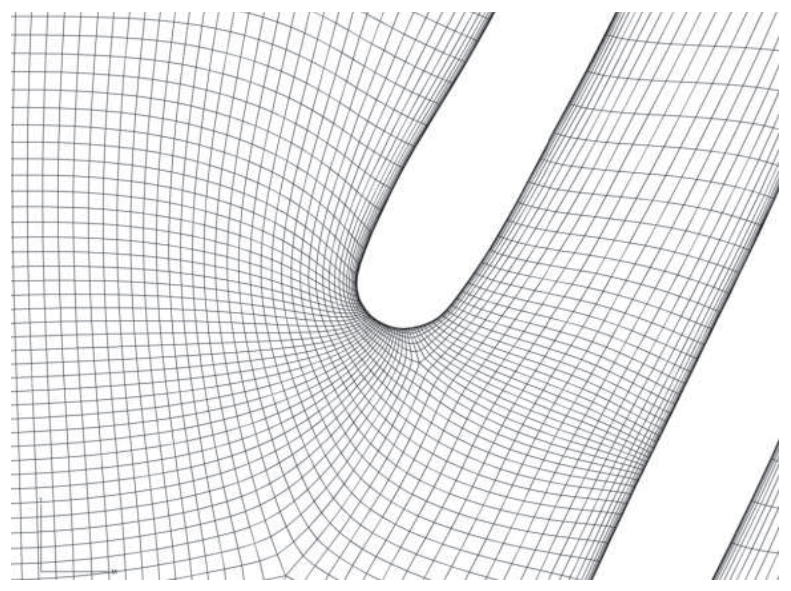

Figure 3. The detail of the impeller blades grid.

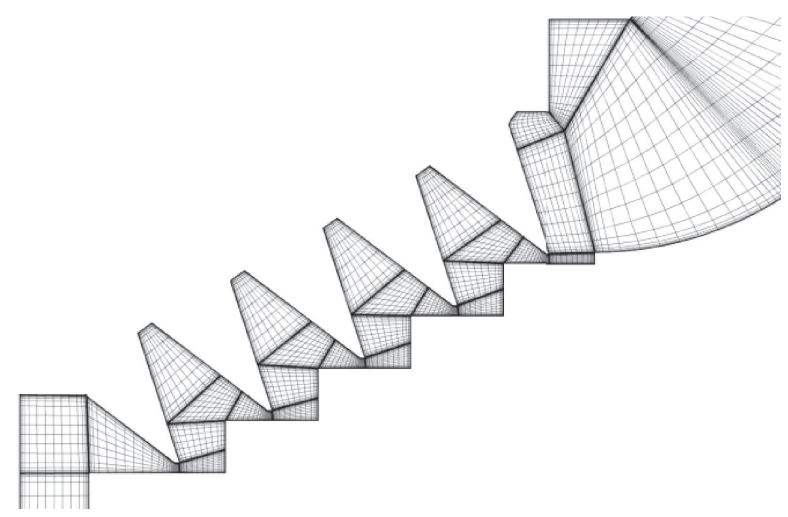

Figure 4. The detail of the stepped seals grid.

Base computational mesh of geometric model with seals and without impeller blades fillets consists approximately of 8000000 hexahedral cells. Thanks to very small height of cells at the walls $(0.003 \mathrm{~mm})$ it was possible to reach maximal $\mathrm{y}+$ around 10 in all solved computations.

\subsubsection{Base geometric stage model with seals and impeller blades fillets}

In case of the geometry model variant with seals and impeller blades fillets wasn't possible to keep automatically generated mesh blocks structure because of higher model complexity. The mesh generator is able to create hub and shroud fillets only at rounded blades, but in our geometry model there is blunt trailing edge on the impeller blades.

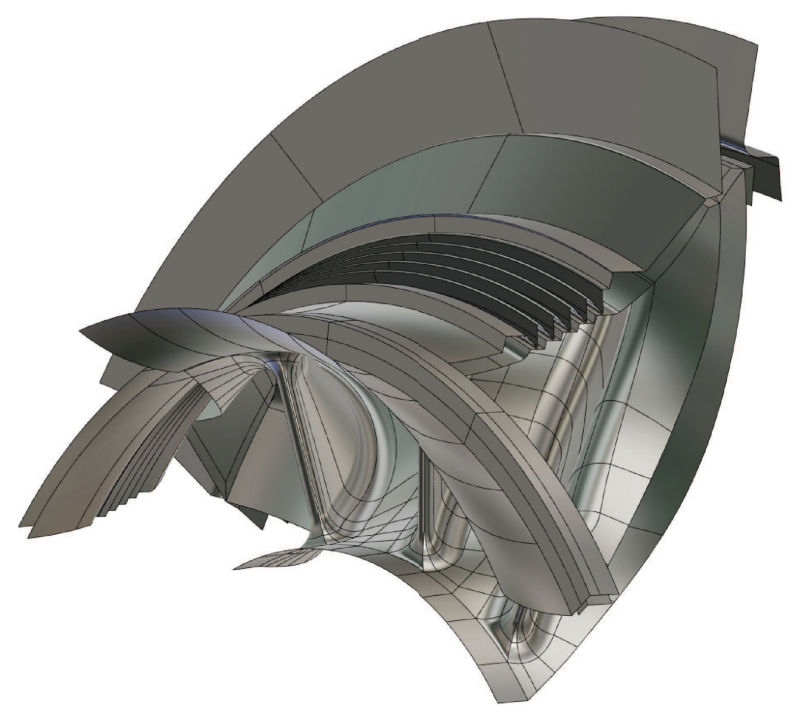

Figure 5. The stage model with impeller hub and shroud fillets.

Due to that we had to create the computational mesh in the impeller area manually so the blocks structure is totally different there, but we was able to keep the same mesh quality parameters as in the case of the automatically generated stage mesh. This mesh consists of 12320000 hexahedral cells.

\subsection{Hub and shroud fillets presence influence}

Solved stage was modeled with the pressure inlet boundary condition on the stage inlet and the mass flow outlet boundary condition on the outlet from the return channel. The boundary between specific functional model areas are defined as the mixing plane interface. Thanks to that we were able to reduce the number of blades to one in the each compressor stage area.

Pressurized medium is in our case air - ideal compressible gas. The SST k- $\omega$ turbulence model was used and cases were computed stationary.

The operational range of the compressor stage is defined its characteristics, where the most important parameters are values of the efficiency and the pressure ratio depending on the mass flow of the pressurized gas through the stage. 


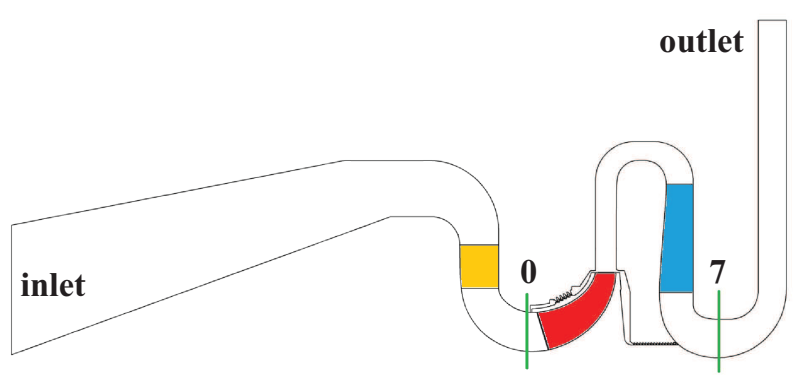

Figure 6. Evaluation planes.

In following graphs are shown working characteristics of simulated stages depending on the mass flow coefficient.

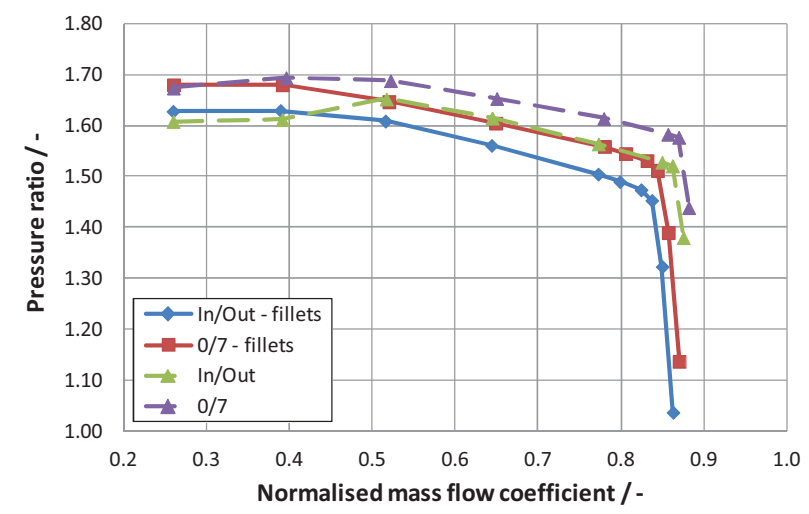

Figure 7. The compressor stage pressure ratio.

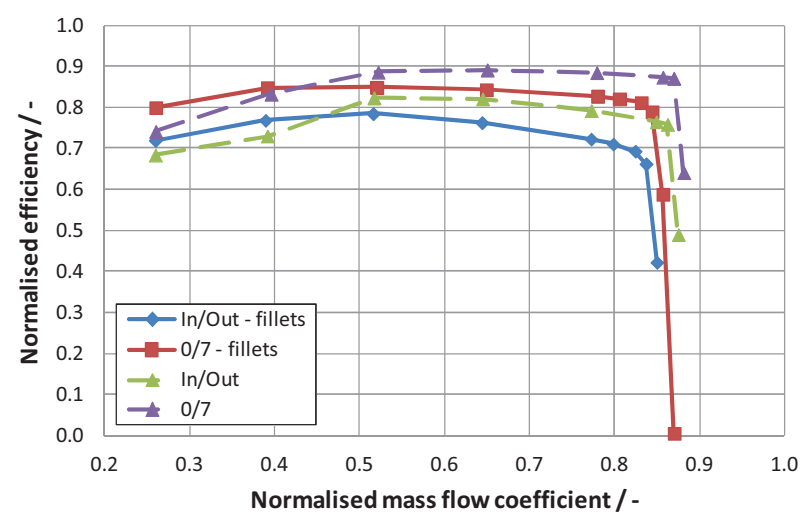

Figure 8. The compressor stage efficiency.

From shown characteristics is obvious that we evaluated the pressure ratio and the isoentropic efficiency in two different sets of evaluating planes. The first evaluated area is between planes "inlet" and "outlet" of the whole model and the second area covers only the impeller and the return channel between planes " 0 " and "7".

As we can see the efficiency decrease in the case of the whole model is approximately $5 \%$ in comparison to the evaluation from planes " 0 " and "7" and it is in accordance with the pressure ratio decrease.

Also the presence of the impeller blades hub and shroud fillets is presented as efficiency and pressure ratio decrease too. In addition to that whole working characteristics are moved to the left and the choke point appears earlier.

\section{Measurement}

A new single radial compressor stage test facility with the 1.2 MW variable speed motor was build to test new designs stages and to compare results from CFD simulations with measured values.

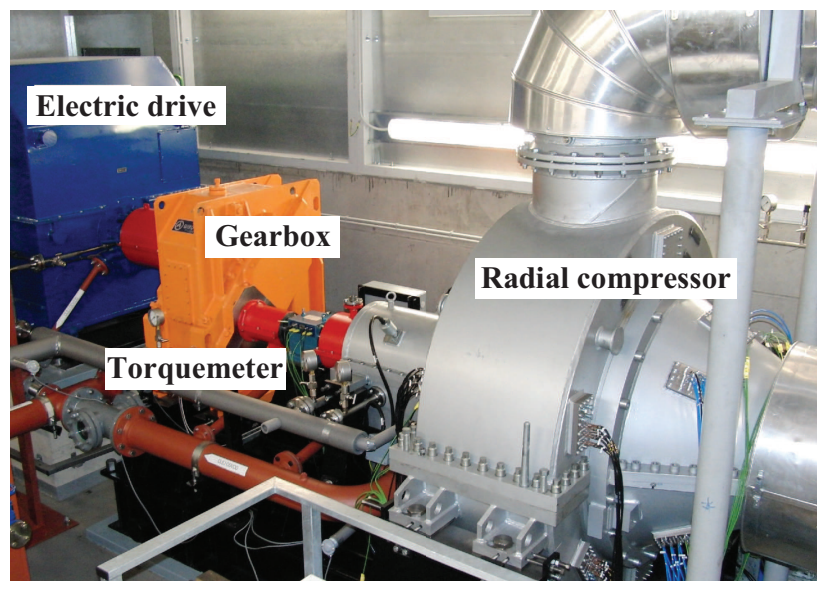

Figure 9. The single radial compressor stage test facility.

The driving electric motor and accessories (cooling system, oil system) are controlled by the complex control system. The tested stages are supplied with measurements to obtain all requested parameters. The mass-flow rates of the air trough the inlet and outlet piping are measured by orifices.

The torquemeter gives the value of the torque and rotating speed of the compressor. The flow parts of the compressor are equipped by tens of probes.

The probes with multiple sensors of the total pressure and total temperature are used in inlet and outlet sections of the stages, in the diffuser and return channel are used single probes of total values. The static pressure samplings are situated in all flow part of the machine.

The built-in probes are complemented by two threehole pressure probes on positioning system and measuring of vanes inlet angles.

The obtained results can be used to various purposes e. g. to the verification of the return vanes design, to the verification of values from built-in probes and to the comparison of measured values with results from CFD simulations.

\subsection{Working characteristics}

In following pictures is shown the comparison of measured pressure ratio and efficiency of the real radial compressor stage with computed values from CFD simulations solved in CFX and NUMECA software and the $1 \mathrm{D}$ design tool. 


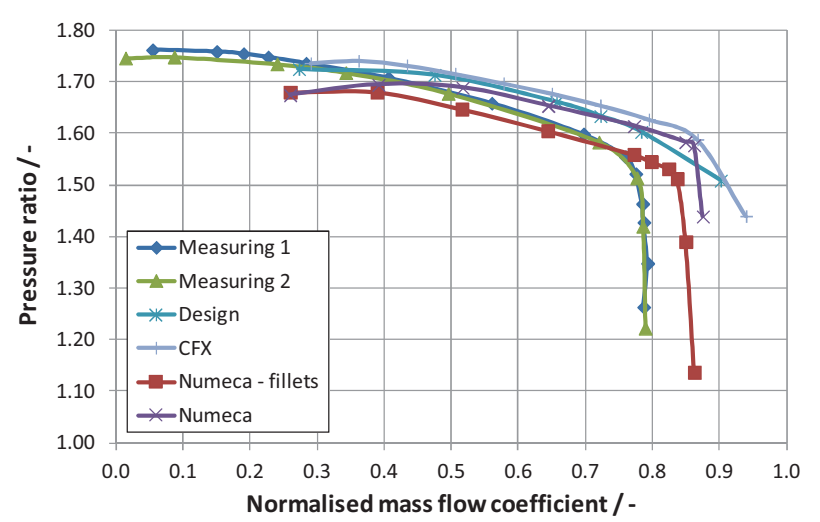

Figure 10. The comparison of CFD sim. with measurement.

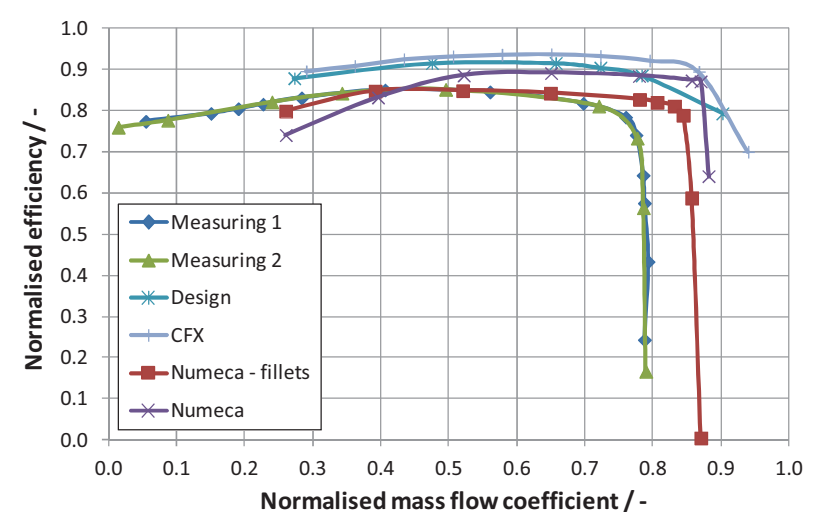

Figure 11. The comparison of CFD sim. with measurement.

When we look on results it's clear that all numerical methods used to obtain working characteristics are limited by the general properties of compressing process. When the pressure ratio is high enough, the flow field in compressor stages becomes unstable so CFD simulations cannot be used near the surge point. This area is possible fully cover only by measuring on the real machine because of rotating stall which appears. Another solution would be full $360^{\circ}$ mesh unsteady simulation but this would be really time consuming. But still there is often large area on working characteristic where compressors work until the surge appears and is possible to compare measured data with computed CFD data. This area is commonly used for impellers CFD design.

From graphs is obvious that there are certain differences of efficiency and pressure ratio in the same working point. CFD simulations without blade fillets give better results in the term of pressure ratio and efficiency values. These ideal working characteristics are also moved to the right so it may seem that compressor stage can work with higher mass flow but this is caused by geometry simplifications.

When the more realistic geometry model with impeller blades fillets was used new working characteristics were closer to measured ones. This is caused by the smaller impeller channel flow section and more realistic flow field but new working characteristics are still moved to the right from measured values and we still investigate main reasons.

\subsection{Inlet angles in front of return vanes}

In the following pictures is shown comparison of inlet angles in front of return channel vanes. Again measured and computed values are compared. In this case differences in results are bigger, but it could be caused by averaging on rotor-stator interfaces and developing measuring methodology.

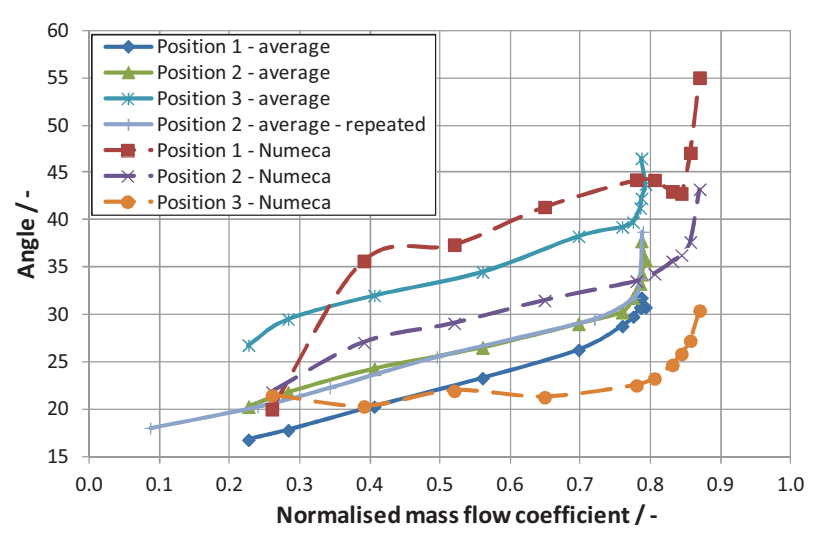

Figure 12. The comparison of evaluated inlet flow angle.

\section{Conclusions}

In this article was mainly investigated the influence of impeller blades hub and shroud fillets presence in the geometrical model on the pressure ratio and the efficiency characteristic of the radial compressor stage.

From evaluated results is obvious that the geometry model with seals and impeller blades hub and shroud fillets gives the closest results to the measurement. There will be always difference between measured data and data computed by CFD. Our results show us that inclusion of the impeller blades fillets into the geometrical model decreases this difference.

\section{Acknowledgments}

The results were developed within the CENTEM project, reg. no. CZ.1.05/2.1.00/03.0088, co-funded by the ERDF as part of the Ministry of Education, Youth and Sports OP RDI programme and in the framework of the FR-TI3/421 project (TIP programme, Ministry of Industry and Trade of the Czech Republic) and specific research.

\section{References}

1. P. C. Hanlon, Compressor handbook (McGraw-Hill, USA, 2001)

2. J. Kadrnožka, Lopatkové stroje (CERM, Brno, 2003)

3. J. Kadrnožka, Tepelné turbíny a turbokompresory (CERM Brno, 2004)

4. J. Bečvář a kol., Tepelné turbíny (SNTL, Praha 1968)

5. M. P. Boyce, Principles of Operation and Performance Estimation of Centrifugal Compressors (Dallas, TX., 1993) 